\title{
Vegetation Analysis and Soil Characteristics on Two Species of Genus Achillea Growing in Egyptian Desert
}

\author{
Yasser Ahmed El-Amier*, El-Sayed Fouad El-Halawany, Samia Ali Haroun, \\ Sura Goma Mohamud \\ Department of Botany, Faculty of Science, Mansoura University, Mansoura, Egypt \\ Email: ${ }^{*}$ yasran@mans.edu.eg
}

Received 29 July 2015; accepted 14 September 2015; published 17 September 2015

Copyright (C) 2015 by authors and Scientific Research Publishing Inc.

This work is licensed under the Creative Commons Attribution International License (CC BY). http://creativecommons.org/licenses/by/4.0/

(c) $\underset{\mathrm{EY}}{\mathrm{B}}$ Open Access

\begin{abstract}
The present study provides a vegetation analysis and species distribution at 50 sites, emphasizing the environmental factors that affect species distribution. A total of 74 plant species belonging to 67 genera and related to 23 families of vascular plants are recorded. Asteraceae, Poaceae, Chenopodiaceae, Brassicaceae, Fabaceae and Zygophyllaceae are the largest families, and therophytes (41.89) and chamaephytes $(24.32 \%)$ are the most frequent, indicating a typical desert life-form spectrum. Chorological analysis revealed that 25 of the studied species were Mediterranean taxa, Saharo-Sindian chorotypes, either pure or penetrated into other regions, comprised 47 species. After application of the TWINSPAN and DCA programs, 4 vegetation groups (A-D) were identified, groups A and B were dominated by Achillea santolina, group C was codominated by Zygophyllm coccinum and Launaea spinosa and group D was dominated by Leptadenia pyrotechnica. Groups A and $B$ may represent the vegetation types of the Western Mediterranean coast of Egypt, while groups C and D may represent the Wadi Hagul. The linear correlation of soil variables with the importance values of some dominant species and the application of Canonical Correspondence Analysis (CCA-biplot) indicates significant associations between the floristic composition of the studied area and the edaphic factors such as electrical conductivity, $\mathrm{pH}$, calcium carbonate, sulphates, bicarbonate, cations $\left(\mathrm{Na}^{+}, \mathrm{K}^{+}, \mathrm{Ca}^{++}\right.$and $\left.\mathrm{Mg}^{++}\right)$and PAR.
\end{abstract}

\section{Keywords}

Achillea, Soil Analysis, Western Mediterranean Coast, Wadi Hagul, Chorotype

${ }^{*}$ Corresponding author. 


\section{Introduction}

The vegetation in Egyptian desert is the most important and characteristic type of natural plant life. Only about $4 \%$ of Egypt's total area is agricultural land and this area has one of the highest population densities in the world. It has been seen from this perspective; reclamation of the desert appears "natural", almost inevitable regarding the population growth and the increased congestion in the old lands which are the lands in the Nile Valley and the Nile Delta [1].

Coastal areas are usually rich in their natural resources that provide great opportunities for economic activities, especially resource-based economic activities such as agriculture, fisheries, tourism, oil and gas production, and maritime transport that tends to locate in these areas [2] [3].

The richest part of Egypt in its floristic composition is the Western Mediterranean coastal belt. This is due to its relatively high rainfall. The number of species in this strip represent about $50 \%$ of the total Egyptian flora which is determined to be about 2000 [4], about 2080 species [5], 2094 species by Boulos [6]. Boulos [7] recorded 2125 species among which 50 species are cultivated. Most of these species are therophytes that appears during the rainy season, giving the coastal belt a temporary showy grassland desert. Vegetation is too sparse to allow meaningful socio-economic activity. Thus, the small number of population in the neighborhood is nomadic, supported economically by breeding and grazing small herds, camels in particular. The environmental effects on vegetation changes are important for both of local and regional management proposes [8].

Egypt's desert is the Sahara, which stretches across much of northern Africa. The few plants that have adapted to the Sahara rely on water retention and protection from animals in the form of spines or toxins. Although the Sahara encompasses multiple countries, certain plants are found only around Egypt and have had important uses or symbolic meanings [9].

Asteraceae (Compositae) is one of the largest families of flowering plants occurring commonly in the world particularly in semiarid region of the tropics and subtropics with about 1600 genera and 25000 species in the world. In the flora of Egypt, Asteraceae is well represented by 92 genera and 226 species. The most members are evergreen shrubs or subshrubs or perennial rhizomatous herbs; biennial and annual herbs are also frequent [10]. On the other hand, Boulos [11] reported that in Egypt, Asteraceae is represented by about 228 species in 98 genera.

The genus Achillea comprises more than 120 species. It is a perennial herb of the family Asteraceae, leaves alternate, pinnatisect, lobed, rarely entire. Achillea fragrantissima (Forssk) Sch. Bip. White-woolly strongly aromatic low shrub, $40-80 \mathrm{~cm}$. Old stems woody, much-branched from the base, flowering branches numerous, herbaceous, terete, rigid, densely woolly. Achillea santolina L. Greyish-woolly perennial herb, $10-30 \mathrm{~cm}$, stems branched, erect or ascending. It occurs in the oases of western desert, the Mediterranean coastal strip, deserts and Sinai Peninsula [10]. The present study aims to investigate relationship between soil variables and wild communities of Achillea fragrantissima and Achillea santolina in the inland and coastal desert of Egypt.

\section{Study Area}

Mariut coast (The western section) extends from Sallum to Abu Qir for about $550 \mathrm{~km}$ (Figure 1). It is described as a thin strip of land parallel to the Mediterranean Sea that widens or narrows according to the position of its southern boundary - the Western Desert Plateau. From sea landward, its average north-south width is about 20 $\mathrm{km}$ and it is bordered by Lake Mariut on the east. The Eastern Desert of Egypt occupies the area extending from the Nile Valley eastward to the Gulf of Suez and the Red Sea. Wadi Hagul is found in the northern part of the Galala Desert (Eastern Desert) of Egypt which extends east of the Nile Delta. On the other hand, Wadi Hagul is found in the valley depression between Gebel Ataqa to the north and the Kahaliya ridge to the south. The channels of this wadi extend for about $35 \mathrm{Km}$ and collects drainage on both sides as well as debouch into the Gluf of Suez. It is described by local physiographic variations and physiognomic heterogeneity [8].

The bioclimatic map of UNESCO/FAO [12] showed that The Mediterranean coastal land of Egypt belongs to the dry arid climatic. The annual mean maximum temperatures range between $25.3^{\circ} \mathrm{C}$ and $23.8^{\circ} \mathrm{C}$ and the annual mean minimum between $13.3^{\circ} \mathrm{C}$ and $15.1^{\circ} \mathrm{C}$. The mean relative humidities are: $67 \%-74 \%$ and $59 \%-71 \%$ in summer and winter respectively [13]. Rainfall occurs during the October-March period (60\% or more); summer is normally dry. The maximum amount falls during either January or December up to $120.8 \mathrm{~mm}$. On the other hand, the climate of the Red Sea coastal land (Wadi Hagul) of Egypt is arid. Temperature is high and ranges between $14^{\circ} \mathrm{C}$ and $21.7^{\circ} \mathrm{C}$ in winter and $23.1^{\circ} \mathrm{C}-46.1^{\circ} \mathrm{C}$ in summer. Relative humidity ranges from $43 \%$ in summer 


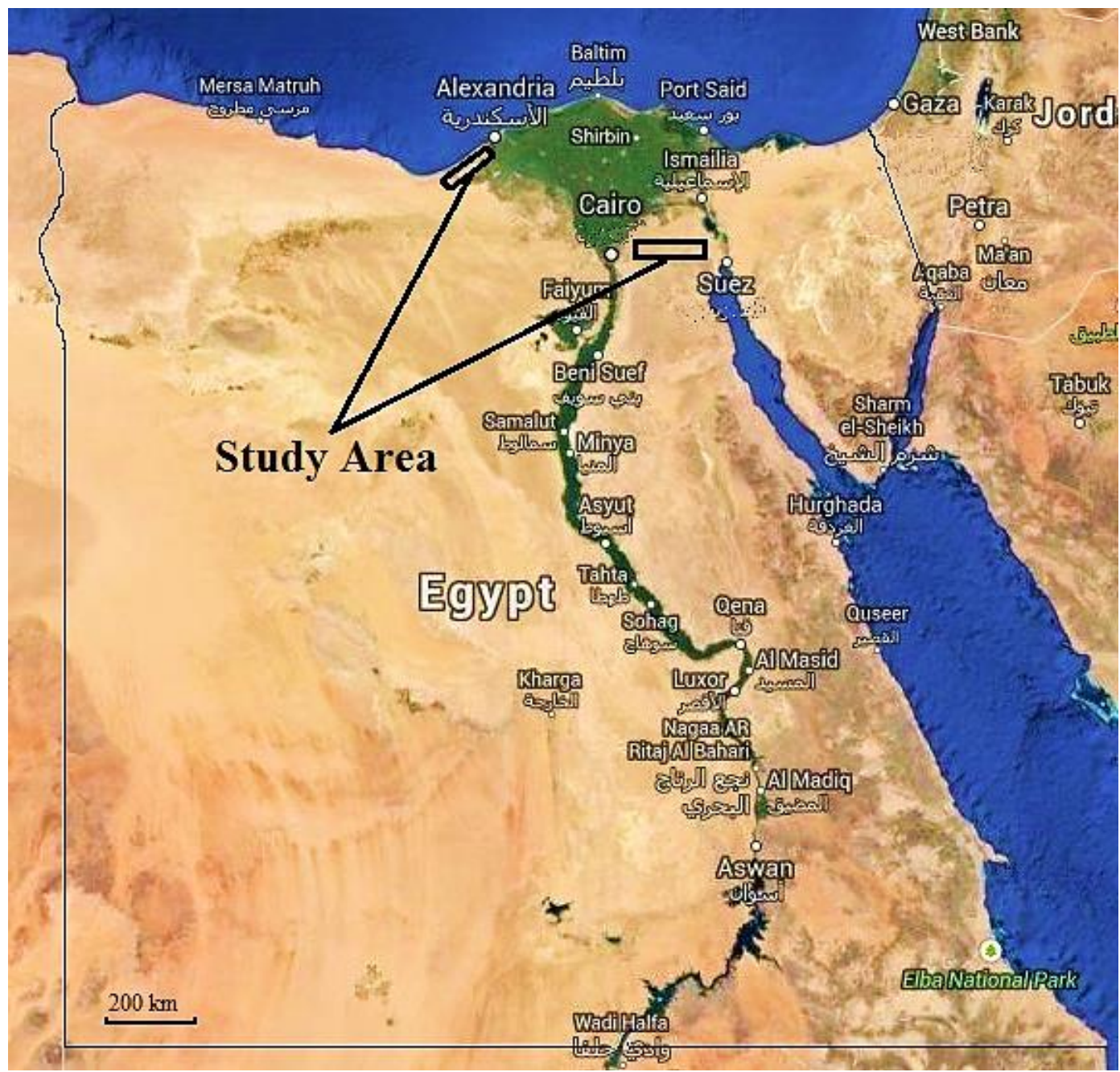

Figure 1. Map of Egypt showing different localities of the study area.

to $65 \%$ in winter. The mean annual rainfall ranges from $25 \mathrm{~mm}$ in Suez to $3.4 \mathrm{~mm}$ in Qusseir. The main amount of rain occurs in winter and summer is, in general, rainless. Variability of annual rainfall is not unusual (55 - 56 $\mathrm{mm})[14][15]$.

\section{Materials and Methods}

\subsection{Vegetation Analysis}

Fifty stands (area $=10 \times 10 \mathrm{~m}$ each) have been selected for sampling vegetation during 2014 as follows: 20 stands in Western Mediterranean coastal belt and 30 in inland desert (Wadi Hagul) of Egypt. In each stand, the annual and perennial species were listed. The nomenclature, identification and floristic categories of plant species were according to Tackholm [5] and up to date by Boulos [7]. Life forms were identified according to the scheme of Raunkiaer [16].

Measuring the density of each plant species was carried out by counting randomly the number of individuals of the species [17]. The plant cover of each species in the studied stands was determined by using the line intercept method according to Canfield [18]. Relative values of cover and density were calculated and estimate of its importance value $(I V=200)$ for each plant species in each stand.

$$
\text { Relative density }=\frac{\text { Absolute density of each species }}{\text { Total absolute density of all species }} \times 100
$$




$$
\text { Relative cover }=\frac{\text { Absolute coverage of a species }}{\text { Total absolute coverage of all species }} \times 100
$$

\subsection{Soil Analysis}

Three soil samples $(0-30 \mathrm{~cm})$ were collected from each stand. The soil samples were pooled together to form one composite sample. They were spread, air dried, sieved (2 $\mathrm{mm}$ sieves) and finally packed in plastic bags to be ready for analysis. Physical and chemical analyses of soil samples were carried out according to Piper [19], Jackson [20] and Allen et al. [21].

\subsection{Data Treatment}

The classification technique used were the Two Way Indicator Species Analysis (TWINSPAN) and Detrended Correspondence Analysis (DCA) applied for the classification of stands into groups and ordinate stands in twodimensional space based on the importance values of species [22]. The relation between the vegetation and soil gradients was assessed using Canonical Correspondence Analysis (CCA) [23] [24]. Data of the soil variables of the vegetation groups identified by TWINSPAN were compared by one-way ANOVA.

\section{Results}

\subsection{Floristic Composition}

The recorded plant species (74) in the present study belonging to 67 genera and related to 23 families, are classified into three major groups as follows: 42 perennials (56.75\%), 31 annuals (41.89\%) and one species biennials (1.35\%) (Table 1). The highest number of species (57) is recorded in the inland desert representing about (77.03\%) of the total recorded species and the coastal desert is represented by 27 species (36.49\%). Table 1 showed that, the family Asteraceae (17 species), Poaceae (9 species), Chenopodiaceae (7 species), Brassicaceae (5 species), Fabaceae (5 species) and Zygophyllaceae (5 species) are represented collectively by 48 species (64.86\% of the total number of the recorded species).

According to Raunkiaer [16], the species are grouped under six types (Figure 2(a)) as follow: Therophytes (31 species $=41.33 \%)$, Chamaephytes $(18$ species $=25.33 \%)$, Hemicryptophytes $(14$ species $=17.33 \%)$, Phanerophytes $(8$ species $=10.67 \%)$, Geophytes $(3$ species $=4.00 \%)$ and Helophytes $($ one species $=1.33 \%)$.

Chorological analysis of the study area revealed that, 23 species (31.08\%) of the total recorded species are Saharo-Sindian. Twenty five species are Mediterranean taxa. It has been also found that, 5 species (6.76\%) are Cosmopolitan, 8 species each (10.81\%) are Saharo-Sindian and Sudano-Zambezian as well as Irano-Turanian and Saharo-Sindian. Pantropical, Polaeotropical, Neotropical and Sudano-Zambezian are represented by one species each (Figure 2(b)).

In the present study, the most common perennial species associated with Achillea fragrantissima communities are: Launaea nudicaulis, Lycium shawii, Artemisia judaica, Crotalaria aegyptiaca, Deverra tortuosa, Diplotaxis harra, Fagonia mollis, Farsetia aegyptia, Gypsophila capillaris and Haloxylon salicornicum. While, the most common perennial species associated with Achillea santolina are: Echinops spinosus, Launaea nudicaulis, Lycium shawii, Atractylis carduus, Convolvulus arvensis, Cynanchum acutum, Fagonia cretica.

\subsection{Classification of Stands}

The application of TWINSPAN classification based on the importance values of 74 plant species recorded in 50 sampled stands representing different habitat types of the study area, led to the recognition of four vegetation groups (Figure 3 and Table 1) and the soil variables are presented in Table 2.

Group A comprises 14 stands dominated by Achillea santolina which has the highest importance value of this group (IV = 42.11). The other important species which attain relatively high IV are: Cynanchum acutum (indicator species IV = 16.17), Convolvulus arvensis (IV = 14.97), Cynodon dactylon (IV = 14.47), Avena fatua (IV $=12.54)$ and Emex spinosa (IV = 10.77). Conyza dioscorides (IV = 11.71). The soil of this group (A) was characterized by relatively high values of sand, organic carbon, $\mathrm{pH}$, bicarbonates, sulphates and extractable cations $\left(\mathrm{Na}^{+}, \mathrm{K}^{+}, \mathrm{Ca}^{++}\right.$and $\left.\mathrm{Mg}^{++}\right)$. 
Table 1. Mean of the importance values (out of 200) of the recorded species in the different vegetation groups resulting from TWINSPAN classification of the sampling stands in the study area habitats. Per. = perennial; Ann. = annuals; $\mathrm{Bi}=$ biennial; Th. = Therophytes; Ch. = Chamaephytes; H. = Hemicryptophytes; He. = Helophytes; G. = Geophytes; Nph. = Nanophanerophytes; COSM = Cosmopolitan; PAL = Palaeotropical; PAN = Pantropical; NEO = Neotropical; ME = Mediterranean; SA-SI = Saharo-Sindian; IT-TR = Irano-Turanian; ER-SR = Euro-Siberian; S-Z = Sudano-Zambezian.

\begin{tabular}{|c|c|c|c|c|c|c|c|c|c|}
\hline \multirow{2}{*}{ No. } & \multirow{2}{*}{ Species } & \multirow{2}{*}{ Family } & \multirow{2}{*}{ Duration } & \multirow{2}{*}{$\begin{array}{l}\text { Life } \\
\text { form }\end{array}$} & \multirow{2}{*}{$\begin{array}{l}\text { Floristic } \\
\text { category }\end{array}$} & \multicolumn{4}{|c|}{ Vegetation groups } \\
\hline & & & & & & A & B & $\mathrm{C}$ & $\mathrm{D}$ \\
\hline \multicolumn{3}{|c|}{ Total number of sites } & & & & 14 & 6 & 21 & 9 \\
\hline \multicolumn{3}{|c|}{ Total number of species } & & & & 26 & 22 & 52 & 17 \\
\hline \multicolumn{10}{|c|}{ Species present in all groups } \\
\hline 1 & Echium angustifolium Mill. & Asteraceae & Per & $\mathrm{H}$ & $\mathrm{ME}+\mathrm{SA}-\mathrm{SI}$ & 3.03 & 17.05 & 2.69 & 5.91 \\
\hline \multicolumn{10}{|c|}{ Species present in three groups } \\
\hline 2 & Alkanna lehmanii (Tin.) A.DC. & Boraginaceae & Per & $\mathrm{H}$ & $\mathrm{ME}$ & 1.56 & 1.01 & 0.28 & - \\
\hline 3 & Erodium laciniatum (Cav.) Wild. & Geraniaceae & Ann & Th & $\mathrm{ME}$ & 10.77 & 1.16 & 0.99 & - \\
\hline 4 & Lasiurus scindicus Henrad & Poaceae & Per & G & $\mathrm{SA}-\mathrm{SI}+\mathrm{S}-\mathrm{Z}$ & 5.99 & 2.71 & 0.60 & - \\
\hline 5 & Launaea spinosa (Forssk.) Sch.Bip. ex Kuntze. & Asteraceae & Per & $\mathrm{Ch}$ & SA-SI & 3.02 & 3.00 & 4.99 & - \\
\hline 6 & Reseda decursiva Forssk. & Resedaceae & Ann & Th & SA-SI & 3.65 & 1.11 & 0.61 & - \\
\hline 7 & Senecio glaucus L. & Asteraceae & Ann & Th & $\mathrm{ME}+\mathrm{IR}-\mathrm{TR}+\mathrm{ER}-\mathrm{SR}$ & 3.62 & 6.58 & 0.15 & - \\
\hline \multicolumn{10}{|c|}{ Species present in two groups } \\
\hline 8 & Achillea fragrantissima (Forssk.) Sch. Bip. & Asteraceae & Per & $\mathrm{Ch}$ & SA-SI + IR-TR & - & - & 14.20 & 10.30 \\
\hline 9 & Achillea santolina L. & Asteraceae & Per & $\mathrm{Ch}$ & SA-SI + IR-TR & 42.11 & 50.59 & - & - \\
\hline 10 & Bassia muoritaca (L.) Asch. & Chenopodiaceae & Ann & Th & IR-TR + SA-SI & 9.69 & 10.45 & - & - \\
\hline 11 & Calotropis procera (Willd.) R.Br. & Asclepiadaceae & Per & $\mathrm{Ph}$ & SA-SI + S-Z & 8.24 & 9.59 & - & - \\
\hline 12 & Cenchrus biflorus Roxb. & Poaceae & Ann & Th & NEO & 4.63 & 1.15 & - & - \\
\hline 13 & Chenopodium murale L. & Chenopodiaceae & Ann & Th & COSM & & & 3.07 & 6.41 \\
\hline 14 & Conyza dioscorides (L.) Desf. & Asteraceae & Per & $\mathrm{Nph}$ & $\mathrm{SA}-\mathrm{SI}+\mathrm{S}-\mathrm{Z}$ & 14.97 & 13.78 & - & - \\
\hline 15 & Crotalaria aegyptiaca Benth. & Fabaceae & Per & $\mathrm{Ch}$ & SA-SI & 11.71 & 5.07 & - & - \\
\hline 16 & Cynanchum acutum L. & Asclepiadaceae & Per & $\mathrm{H}$ & $\mathrm{ME}+\mathrm{IR}-\mathrm{TR}$ & - & - & 3.51 & - \\
\hline 17 & Cynodon dactylon (L.) Pers. & Poaceae & Per & G & COSM & 16.17 & 8.49 & - & - \\
\hline 18 & Diplotaxis harra (Forssk.) Boiss. & Brassicaceae & Per & Ch & $\mathrm{ME}+\mathrm{SA}-\mathrm{SI}$ & 14.47 & 21.25 & - & - \\
\hline 19 & Emex spinosa (L.) Campd. & Polygonaceae & Ann & Th & $\mathrm{ME}+\mathrm{SA}-\mathrm{SI}$ & 1.94 & 5.47 & - & - \\
\hline 20 & Fagonia mollis Delile. & Zygophyllaceae & Per & Ch & SA-SI & 0.64 & 10.79 & - & - \\
\hline 21 & Iphiona mucronata (Forssk.) Asch. \& Schweinf. & Asteraceae & Per & $\mathrm{Ch}$ & SA-SI & 4.41 & 3.91 & - & - \\
\hline 22 & Haloxylon salicornicum (Moq.) Bunge ex Boiss & Chenopodiaceae & Per & $\mathrm{Ch}$ & SA-SI & - & - & 1.84 & 1.81 \\
\hline 23 & Lycium shawii Roem. \& Schult. & Solanaceae & Per & Nph & $\mathrm{SA}-\mathrm{SI}+\mathrm{S}-\mathrm{Z}$ & - & - & 1.94 & 7.45 \\
\hline 24 & Malva parviflora L. & Malvaceae & Ann & Th & $\mathrm{ME}+\mathrm{IR}-\mathrm{TR}$ & - & 13.01 & 1.47 & - \\
\hline 25 & Mesembryanthemum nodiflorum L. & Aizoaceae & Ann & Th & $\mathrm{ME}+\mathrm{ER}-\mathrm{SR}+\mathrm{SA}-\mathrm{SI}$ & 2.85 & 2.11 & - & - \\
\hline 26 & Neurada procumbense L. & Neuradaceae & Ann & Th & $\mathrm{SA}-\mathrm{SI}+\mathrm{S}-\mathrm{Z}$ & 5.87 & 10.67 & - & - \\
\hline 27 & Panicum turgidum Forssk & Poaceae & Per & $\mathrm{H}$ & SA-SI & - & - & 11.74 & 10.81 \\
\hline 28 & Plantago notato Log. & Plantaginaceae & Ann & Th & IR-TR + SA-SI & - & - & 0.31 & 3.97 \\
\hline 29 & Poa annua L. & Poaceae & Ann & Th & COSM & - & - & 1.47 & 0.63 \\
\hline 30 & Deverra tortuosa (Desf.) DC. & Apiaceae & Per & $\mathrm{Ch}$ & SA-SI & 0.59 & - & 0.55 & - \\
\hline 31 & Reichardia tingitana (L.) Roth. & Asteraceae & Ann & Th & $\mathrm{ME}+\mathrm{IR}-\mathrm{TR}$ & - & - & 1.19 & 11.55 \\
\hline 32 & Retama raetam (Forssk.) Webb \& Berthel. & Fabaceae & Ann & Th & $\mathrm{ME}+\mathrm{SA}-\mathrm{SI}+\mathrm{S}-\mathrm{Z}$ & 2.88 & 1.06 & - & - \\
\hline 33 & Rumex vesicarius L. & Polygonaceae & Per & Nph & $\mathrm{ME}+\mathrm{IR}-\mathrm{TR}+\mathrm{SA}-\mathrm{SI}$ & - & - & 12.48 & 4.58 \\
\hline
\end{tabular}




\section{Continued}

\begin{tabular}{|c|c|c|c|c|c|c|c|c|}
\hline 34 & Volutaria lippii (L.) Cass. Ex Maire & Asteraceae & Ann & $\mathrm{Th}$ & SA-SI & - & -1.94 & 7.32 \\
\hline 35 & Zilla spinosa (L.) prantl & Brassicaceae & Per & $\mathrm{Ch}$ & SA-SI & - & - $\quad 7.14$ & 13.86 \\
\hline 36 & Zygophyllum coccinum L. & Zygophyllaceae & Per & $\mathrm{Ch}$ & SA-SI & - & -23.38 & 22.67 \\
\hline 37 & Zygophyllum decumbns Delile & Zygophyllaceae & Per & $\mathrm{Ch}$ & SA-SI & - & $-\quad 3.36$ & 10.53 \\
\hline \multicolumn{9}{|c|}{ Species present in one group } \\
\hline 38 & Anabasis articulata (Forssk.) Moq & Chenopodiaceae & Per & $\mathrm{Ch}$ & SA-SI + IR-TR & - & - $\quad 0.91$ & - \\
\hline 39 & Artemisia judaica L. & Asteraceae & Per & $\mathrm{Ch}$ & SA-SI & 1.45 & - & - \\
\hline 40 & Astragalus bombycinus Boiss & Fabaceae & Ann & $\mathrm{H}$ & IR-TR + SA-SI & - & $-\quad 1.46$ & - \\
\hline 41 & Atractylis carduus (Forssk.) C.Chr. & Asteraceae & Per & $\mathrm{H}$ & $\mathrm{ME}+\mathrm{SA}-\mathrm{SI}$ & - & -0.75 & - \\
\hline 42 & $\begin{array}{l}\text { Atriplex lindleyi Moq.subsp. Inflata } \\
\text { (F. Muell.) Wilson. }\end{array}$ & Chenopodiaceae & Ann & Th & $\mathrm{ME}+\mathrm{IR}-\mathrm{TR}+\mathrm{ER}-\mathrm{SR}$ & 4.54 & - & - \\
\hline 43 & Avena fatua $\mathrm{L}$. & Poaceae & Ann & Th & PAL & - & -1.55 & - \\
\hline 44 & Bassia indica (Wight) Scott & Chenopodiaceae & Ann & Th & $\mathrm{IR}-\mathrm{TR}+\mathrm{S}-\mathrm{Z}$ & 12.54 & - & - \\
\hline 45 & Brassica tournefortii Gouan & Brassicaceae & Ann & Th & $\mathrm{ME}+\mathrm{IR}-\mathrm{TR}+\mathrm{SA}-\mathrm{SI}$ & - & $-\quad 1.61$ & - \\
\hline 46 & Carthamus tenuis (Boiss \& Blanche) Bornm. & Asteraceae & Ann & Th & $\mathrm{ME}$ & - & -0.83 & - \\
\hline 47 & Centaurea aegyptiaca L. & Asteraceae & $\mathrm{Bi}$ & Th & SA-SI & 8.68 & - & - \\
\hline 48 & Cleome droserifolia (Forssk.) Delile & Cleomeaceae & Per & $\mathrm{Ch}$ & IR-TR + SA-SI & - & -1.36 & - \\
\hline 49 & Convolvulus arvensis L. & Convolvalaceae & Per & $\mathrm{H}$ & COSM & - & $-\quad 2.31$ & - \\
\hline 50 & Echinops spinosus L. & Asteraceae & Per & $\mathrm{H}$ & $\mathrm{ME}+\mathrm{SA}-\mathrm{SI}$ & - & - 7.54 & - \\
\hline 51 & Euphorbia retusa Forssk. & Euphorbiaceae & Ann & Th & SA-SI & - & $-\quad 1.98$ & - \\
\hline 52 & Fagonia cretica L. & Zygophyllaceae & Per & $\mathrm{Ch}$ & ME & - & -2.07 & - \\
\hline 53 & Farsetia aegyptia Turra. & Brassicaceae & Per & $\mathrm{Ch}$ & $\mathrm{SA}-\mathrm{SI}+\mathrm{S}-\mathrm{Z}$ & - & $-\quad 2.84$ & - \\
\hline 54 & Gypsophila capillaris (Forssk.) C. Chr & Caryophyllaceae & Per & $\mathrm{H}$ & IR-TR + SA-SI & - & -2.52 & - \\
\hline 55 & Hordeum leporinum L. & Poaceae & Ann & Th & $\mathrm{ME}+\mathrm{IR}-\mathrm{TR}+\mathrm{ER}-\mathrm{SR}$ & - & -8.54 & - \\
\hline 56 & Ifloga spicata (Forssk.) Sch.Bip. & Asteraceae & Ann & Th & SA-SI & - & $-\quad 1.75$ & - \\
\hline 57 & Imperata cylindrical (L.) Raeusch. & Poaceae & Per & $\mathrm{H}$ & $\mathrm{ME}+\mathrm{PAL}$ & - & -6.37 & - \\
\hline 58 & Lactuca serriola L. & Asteraceae & Ann & Th & $\mathrm{ME}+\mathrm{IR}-\mathrm{TR}+\mathrm{ER}-\mathrm{SR}$ & - & -4.51 & - \\
\hline 59 & Launaea nudicaulis (L.) Hook. f. & Asteraceae & Per & $\mathrm{H}$ & SA-SI & - & -1.52 & - \\
\hline 60 & Lavandula coronopifolia Poir. & Lamiaceae & Per & $\mathrm{Ch}$ & SA-SI & - & - 19.33 & - \\
\hline 61 & Leptadenia pyrotechnica (Forrsk.) Decne. & Asclepiadaceae & Per & Nph & SA-SI & - & - & 22.07 \\
\hline 62 & Lotus glinoides Delile. & Fabaceae & Ann & Th & $\mathrm{S}-\mathrm{Z}$ & - & - & 29.48 \\
\hline 63 & Matthiola longipetala (Vent.) DC. & Brassicaceae & Ann & Th & $\mathrm{ME}+\mathrm{IR}-\mathrm{TR}$ & - & -1.04 & - \\
\hline 64 & Mesembryanthemum crystallinum L. & Aizoaceae & Ann & Th & $\mathrm{ME}+\mathrm{ER}-\mathrm{SR}$ & - & $-\quad 2.86$ & - \\
\hline 65 & Ochradenus baccatus Delile. & Resedaceae & Per & Nph & SA-SI & - & -0.28 & - \\
\hline 66 & Phragmites australis (Cav.) Trin. ex. Steud. & Poaceae & Per & $\mathrm{G}, \mathrm{He}$ & COSM & - & -6.08 & - \\
\hline 67 & Polycarpaea repens (Forssk.) Asch. & Caryophyllaceae & Per & $\mathrm{Ch}$ & SA-SI & - & $-\quad 3.24$ & - \\
\hline 68 & Pulicaria undulata (L.) C. A. Mey. & Asteraceae & Per & $\mathrm{Ch}$ & SA-SI & - & -0.26 & - \\
\hline 69 & Salsola kali L. & Chenopodiaceae & Ann & Th & COSM & - & $-\quad 3.48$ & - \\
\hline 70 & Spergularia rubra (L.) J. \& C. Presl. & Caryophyllaceae & Ann & Th & $\mathrm{ME}+\mathrm{ER}-\mathrm{SR}$ & - & -3.23 & - \\
\hline 71 & Tamarix aphylla (L.) H. Karst. & Tamaricaceae & Per & Nph & SA-SI + S-Z & - & -0.16 & - \\
\hline 72 & Trichodesma africanum (L.) R. Br. & Boraginaceae & Per & $\mathrm{H}$ & SA-SI + S-Z & - & - & 18.82 \\
\hline 73 & Trigonella stellata Forssk & Fabaceae & Ann & Th & IR-TR + SA-SI & - & - & 8.54 \\
\hline 74 & Zygophyllum simplex L. & Zygophyllaceae & Ann & Th & SA-SI & - & - 9.20 & - \\
\hline
\end{tabular}


a- Life form of study area

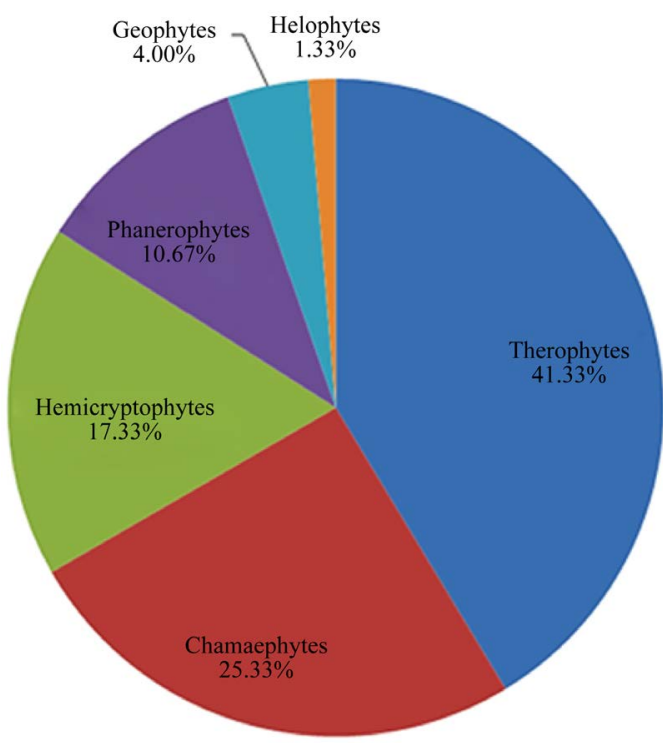

(a) b- Floristic category of study area

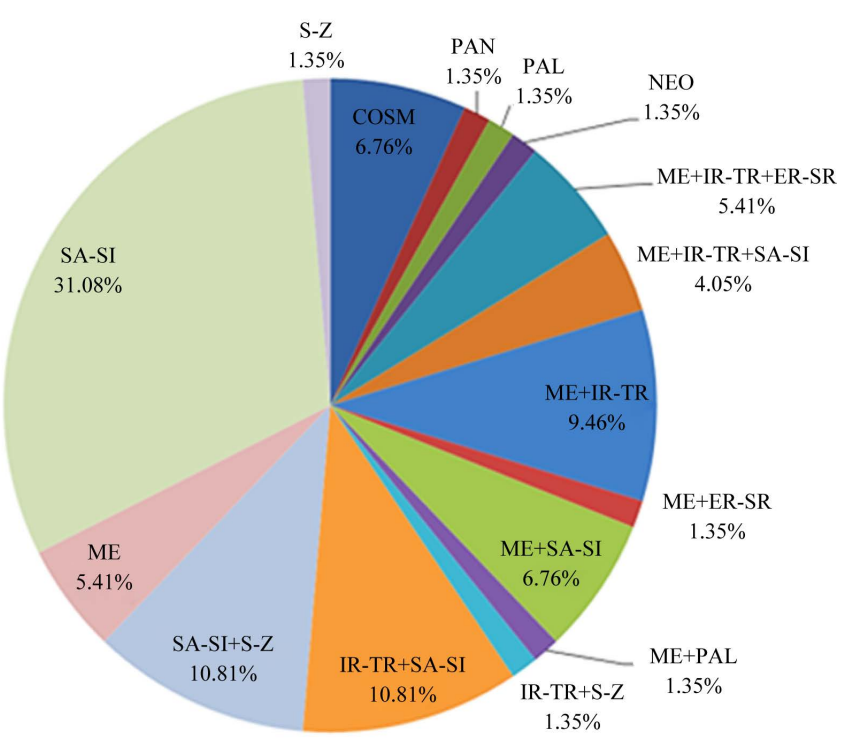

(b)

Figure 2. Diagram of life form (a) and floristic category (b) of the study area.

Table 2. Mean values, standard errors $( \pm \mathrm{SE})$, and ANOVA F-values of the soil variables of the 50 sites representing the 4 vegetation groups (A-D) obtained by cluster analysis. OC: organic carbon; EC: electrical conductivity; SAR: sodium adsorption ratio, PAR: potassium adsorption ratio. $* \mathrm{P}<0.05$, $* * \mathrm{P}<0.01$, ***P $<0.001$.

\begin{tabular}{|c|c|c|c|c|c|c|c|}
\hline \multirow{2}{*}{\multicolumn{2}{|c|}{ Soil variable }} & \multicolumn{3}{|c|}{ Vegetation groups } & \multirow[b]{2}{*}{$\mathrm{D}$} & \multirow{2}{*}{ F-value } & \multirow{2}{*}{$\mathrm{LSD}_{0.05}$} \\
\hline & & A & B & $\mathrm{C}$ & & & \\
\hline Sand & \multirow{7}{*}{$(\%)$} & $94.26 \pm 1.10$ & $93.18 \pm 1.48$ & $87.81 \pm 1.42$ & $91.92 \pm 2.84$ & 1.94 & $6.67^{\mathrm{ns}}$ \\
\hline Silt & & $4.68 \pm 0.98$ & $5.73 \pm 1.23$ & $10.76 \pm 1.31$ & $7.04 \pm 2.68$ & 2.11 & $6.1^{\mathrm{ns}}$ \\
\hline Clay & & $1.06 \pm 0.17$ & $1.08 \pm 0.28$ & $1.43 \pm 0.13$ & $1.04 \pm 0.26$ & 0.35 & $0.85^{\mathrm{ns}}$ \\
\hline Porosity & & $33.28 \pm 1.58$ & $34.54 \pm 0.87$ & $30.54 \pm 1.12$ & $33.21 \pm 1.96$ & 0.88 & $6.81^{\mathrm{ns}}$ \\
\hline WHC & & $28.20 \pm 3.35$ & $26.41 \pm 1.24$ & $26.40 \pm 1.29$ & $32.51 \pm 1.54$ & 0.37 & $12.14^{\mathrm{ns}}$ \\
\hline $\mathrm{CaCO}_{3}$ & & $9.82 \pm 0.45$ & $9.47 \pm 0.88$ & $22.04 \pm 2.43$ & $15.25 \pm 3.96$ & 2.16 & 10.55 \\
\hline OC & & $0.31 \pm 0.07$ & $0.29 \pm 0.07$ & $0.17 \pm 0.02$ & $0.19 \pm 0.04$ & 1.31 & $0.19^{\text {ns }}$ \\
\hline \multicolumn{2}{|r|}{$\mathrm{pH}$} & $8.45 \pm 0.08$ & $8.45 \pm 0.07$ & $8.02 \pm 0.07$ & $8.00 \pm 0.08$ & 10.53 & $0.33^{* * *}$ \\
\hline \multicolumn{2}{|c|}{$\mathrm{EC}(\mu \mathrm{mhos} / \mathrm{cm})$} & $297.38 \pm 61.48$ & $222.50 \pm 44.91$ & $383.37 \pm 72.67$ & $545.25 \pm 125.34$ & 3.87 & $252.58^{*}$ \\
\hline $\mathrm{Cl}^{-}$ & \multirow{4}{*}{ (\%) } & $0.15 \pm 0.01$ & $0.16 \pm 0.02$ & $0.19 \pm 0.10$ & $0.42 \pm 0.20$ & 0.27 & 0.46 \\
\hline $\mathrm{SO}_{4}^{2-}$ & & $0.64 \pm 0.05$ & $0.68 \pm 0.07$ & $0.26 \pm 0.06$ & $0.33 \pm 0.10$ & 5.42 & $0.29 * *$ \\
\hline $\mathrm{CO}_{3}^{2-}$ & & $0.00 \pm 0.00$ & $0.00 \pm 0.00$ & $0.62 \pm 0.15$ & $0.38 \pm 0.20$ & 1.63 & $0.65^{\mathrm{ns}}$ \\
\hline $\mathrm{HCO}_{3}^{-}$ & & $3.10 \pm 0.30$ & $3.53 \pm 0.39$ & $1.22 \pm 0.11$ & $0.61 \pm 0.14$ & 20.95 & $0.88 * * *$ \\
\hline $\mathrm{Na}^{+}$ & \multirow{4}{*}{ mg/100 dry soil } & $676.31 \pm 128.14$ & $489.00 \pm 69.86$ & $204.81 \pm 52.12$ & $101.45 \pm 60.03$ & 10.04 & $195.74 * * *$ \\
\hline $\mathrm{K}^{+}$ & & $327.69 \pm 57.62$ & $240.67 \pm 29.15$ & $24.53 \pm 5.43$ & $15.22 \pm 6.89$ & 45.44 & $55.73^{* * *}$ \\
\hline $\mathrm{Ca}^{++}$ & & $2383.77 \pm 621.98$ & $1675.33 \pm 246.46$ & $66.41 \pm 15.80$ & $42.82 \pm 23.37$ & 34.15 & $457.96^{* * *}$ \\
\hline $\mathrm{Mg}^{++}$ & & $641.15 \pm 122.58$ & $478.83 \pm 65.91$ & $30.74 \pm 7.01$ & $13.20 \pm 7.34$ & 43.49 & $114.93^{* * *}$ \\
\hline \multicolumn{2}{|r|}{ SAR } & $17.10 \pm 1.33$ & $14.72 \pm 1.06$ & $25.93 \pm 3.54$ & $16.48 \pm 5.29$ & 0.25 & $13.73^{\text {ns }}$ \\
\hline \multicolumn{2}{|r|}{ PAR } & $8.42 \pm 0.53$ & $7.32 \pm 0.36$ & $3.28 \pm 0.31$ & $2.78 \pm 0.43$ & 28.96 & $1.42^{* * *}$ \\
\hline
\end{tabular}


Group B comprises 6 stands dominated by Achillea santolina which has the highest importance value (IV = 50.59). The other important species which attain relatively high IV are: Cynodon dactylon (IV = 21.25), Echinops spionousus (IV = 17.05), Convolvulus arvensis (IV = 13.78), Lycium shawii (IV = 13.01), Mesembryanthemum nodiflorum (IV = 10.67) and Bassia indica (IV = 10.45). The indicator species in this group is Imperata cylindrica (IV = 3.91). The soil was characterized by high percentages of porosity, $\mathrm{pH}$, organic carbon, bicarbonates, sulphates, moderate value of chlorides and extractable cations $\left(\mathrm{Na}^{+}\right.$and $\left.\mathrm{Ca}^{++}\right)$.

Group C comprises 21 stands codominated by Zygophyllum coccinum (IV = 23.38) and Launaea spinosa (IV $=19.33$ ). The other important species which attain relatively high IV are: Achillea fragrantissima (IV = 14.20), Retama raetam (IV = 12.48), Ochradenus baccatus (IV = 11.74). The indicator species in this group are: Panicum turgidum (IV = 6.08), Crotalaria aegyptiaca (IV = 3.511) and Echinops spinosus (IV = 2.69). The soil of this group was characterized by high content of soil fractions (silt \& clay), calcium carbonate and carbonate, while, this soil attained moderate values of electrical conductivity, chloride, sodium and SAR.

Group D comprises 9 stands dominated by Leptadenia pyrotechnica (indicator species IV $=29.48$ ). The other important species which attain relatively high IV are: Zygophyllum coccinum (IV = 22.67), Lavandula coronopifolia (IV = 22.07) and Tamarix aphylla (indicator species IV = 18.82), Zilla spinosa (IV = 13.86), Ochradenus baccatus (IV = 10.81) and Achillea fragrantissima (IV = 10.30). The soil was characterized by high content of sand, water-holding capacity, electrical conductivity and chloride, moderate value of calcium carbonate, carbonates and sulphates.

\subsection{Ordination of Stands}

The ordination of stands in the different habitats of the study area, given by Detrended Correspondence Analysis (DCA) is demonstrated in Figure 4. The DCA ordination of stands is shown on the plane of the first and second DCA axes. It is obvious that, the groups of vegetation obtained by TWINSPAN classification are remarkably distinguishable and having a clear pattern of segregation on the ordination plane.

Groups A and B dominated by Achillea santolina are separated at the right side of the DCA diagram and showed also superimposed intercept. On the other hand, group C codominated by Zygophyllum coccinum and Launaea spinosa is separated at the middle part of the DCA diagram. Group D dominated by Leptadenia pyrotechnica is obviously separated at the upper left side of the DCA diagram.

\subsection{Vegetation-Soil Relationships}

The variation in soil factors of the four vegetation groups of stands resulting from TWINSPAN classification indicated considerable variations in the edaphic factors among the stands of the different groups (Table 2). Electrical conductivity, $\mathrm{pH}$, sulphates, bicarbonate, cations $\left(\mathrm{K}^{+}, \mathrm{Na}^{+}, \mathrm{Ca}^{++}\right.$and $\left.\mathrm{Mg}^{++}\right)$and PAR showed significant correlations ( $\mathrm{P}<0.05)$ among vegetation groups. The highest percentage of coarse fractions (sand $=94.26 \%$ ) was obtained in group A, but the highest percentage of silt (10.76\%) and clay (1.43\%) fraction were obtained in group C. Vegetation groups C and D showed higher values of electrical conductivity (383.37 and 545.25 $\mu \mathrm{mohs} / \mathrm{cm}$, respectively) than in groups A and B (297.38 and $222.50 \mu \mathrm{mohs} / \mathrm{cm}$, respectively). Also, the percentages of sulphates and bicarbonate were relatively higher in groups A and B $(0.64 \%, 0.68 \%$ and $1.27 \%, 3.53 \%$, respectively) as compared with groups $\mathrm{C}$ and $\mathrm{D}(0.26 \%, 0.33 \%$ and $1.22 \%, 0.61 \%$, respectively). $\mathrm{pH}$ was higher in groups A and B (8.45 each) than in groups $C$ and D (8.0 each). Vegetation groups A and B showed values of cations and PAR which were higher than in groups $\mathrm{C}$ and $\mathrm{D}$.

Correlations of edaphic variables with the importance values of the dominant and abundant species are shown in Table 3. It has been found that, some soil variables are positively correlated with plant species such as Achillea fragrantissima correlated significantly with silt $(r=0.392)$ and carbonate calcium $(r=0.415)$. Achillea santolina showed high ( $r=0.375,0.403,0.633,0.629,0.758,0.568,0.753,0.661,0.745$ and 0.814 ) significant correlations with sand, organic carbon, $\mathrm{pH}, \mathrm{SO}_{4}, \mathrm{HCO}_{3}, \mathrm{Na}, \mathrm{K}, \mathrm{Ca}, \mathrm{Mg}$ and $\mathrm{PAR}$, respectively. Cynodon dactylon and Cynanchum acutum were correlated significantly with all edaphic factors except soil texture, porosity, WHC, organic carbon, electrical conductivity and chloride. Calcium carbonate and electrical conductivity were correlated significantly with Zygophyllum coccinum $(r=0.575)$ and Tamarix aphylla $(r=0.393)$. On the other hand, it has been also found that, some soil variables such as clay, porosity, $\mathrm{WHC}, \mathrm{CO}_{3}$ and SAR have negative correlated or no correlation with plant species. 


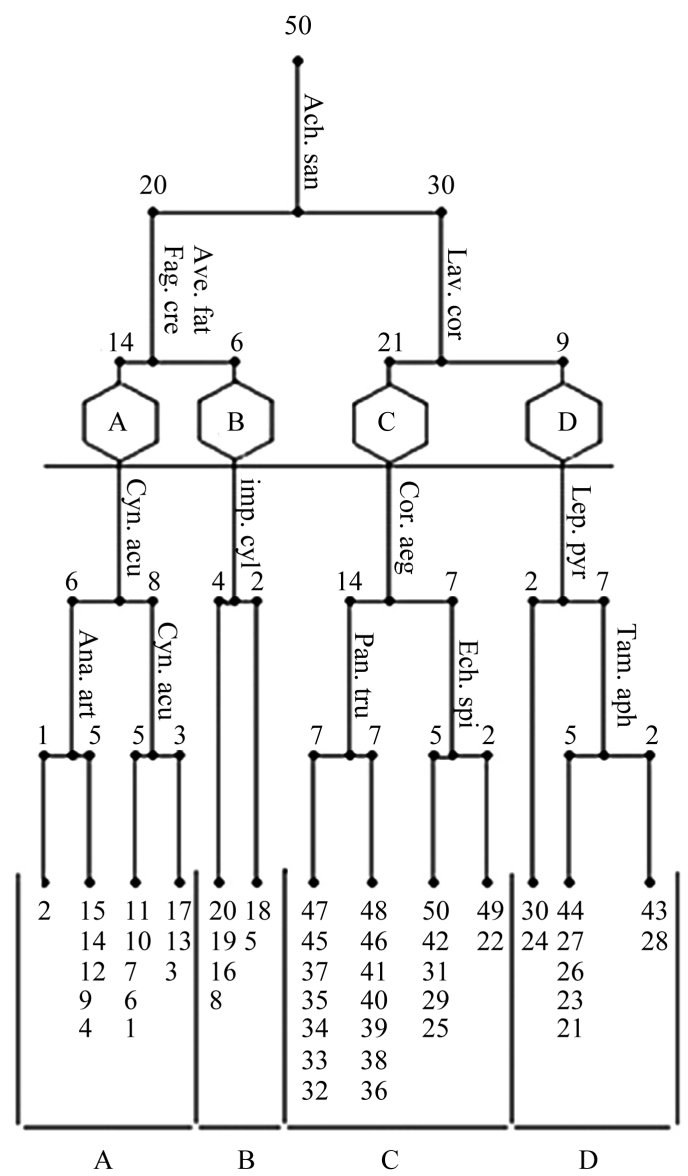

Figure 3. Two Way Indicator Species Analysis (TWINSPAN) dendrogram of the 50 sampled stands based on the importance values of the 74 species. The indicator species are abbreviated by the first three letters of genus and species, respectively.

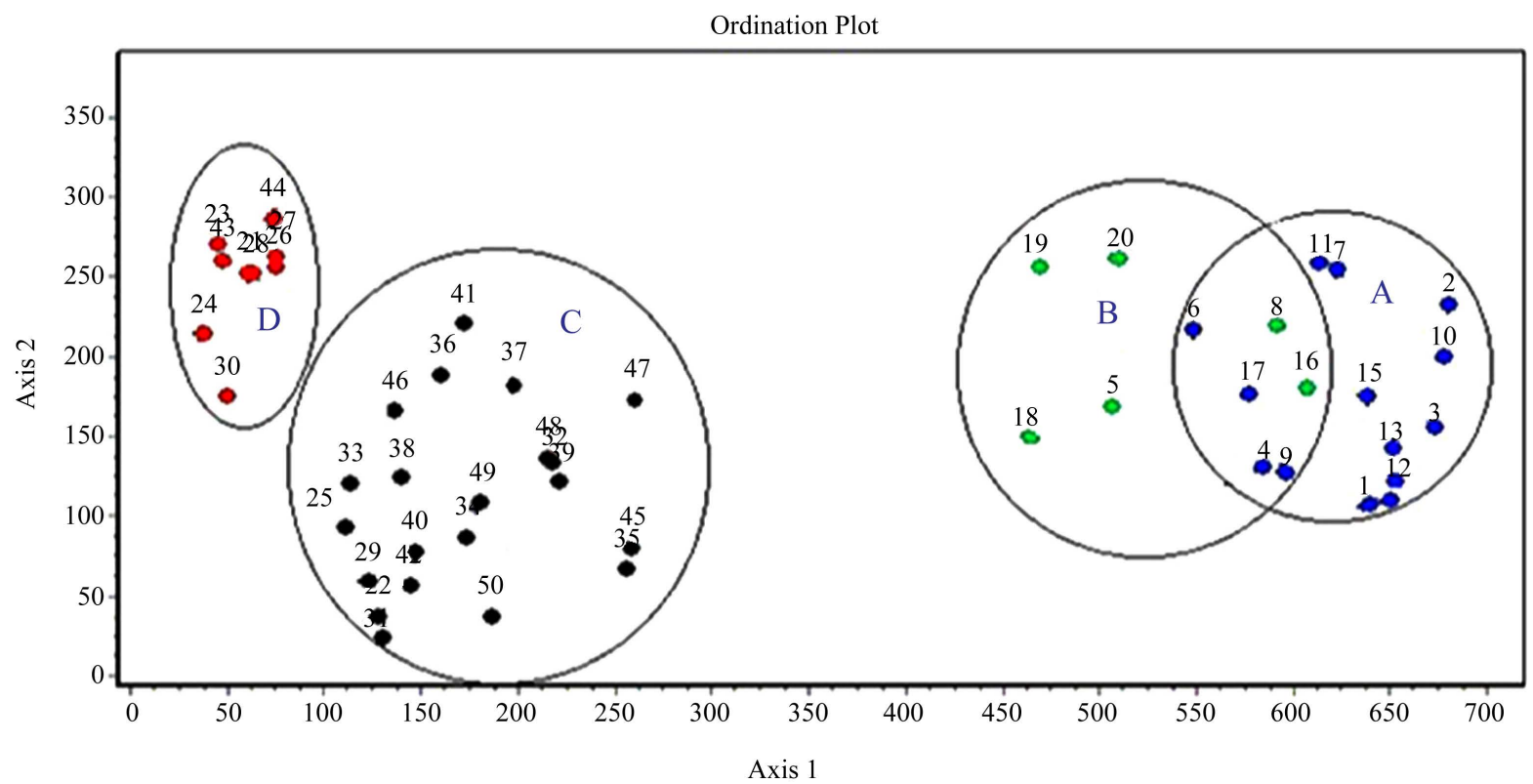

Figure 4. Detrended Correspondence Analysis (DCA) ordination diagram of the 50 stands with vegetation groups. 


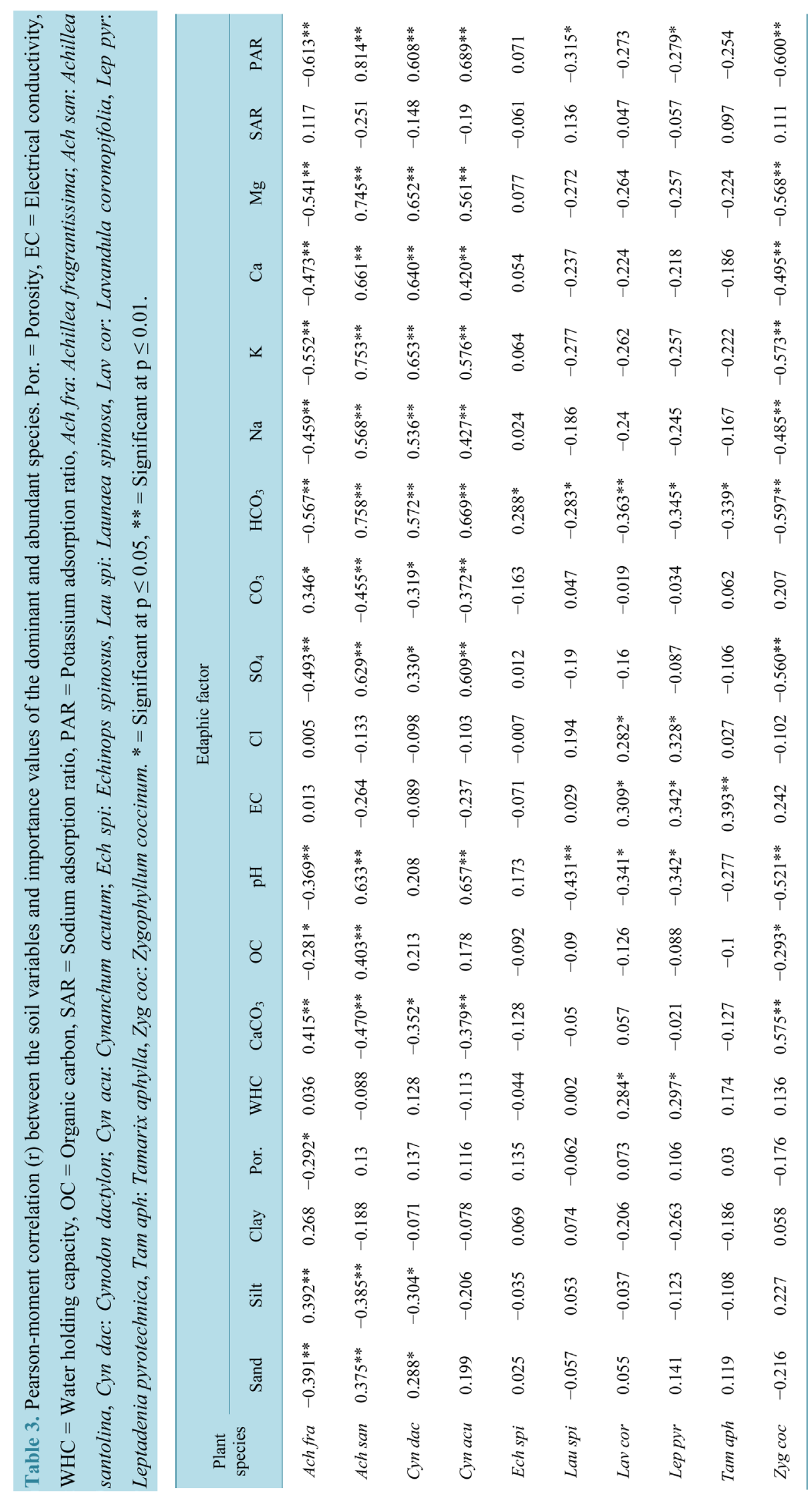


The correlation between vegetation and soil variables is indicated on the ordination diagram produced by Canonical Correspondence Analysis (CCA) of the biplot of species and environmental variables. As shown in Figure 5, the electrical conductivity, sulphate, calcium carbonate, $\mathrm{pH}$, potassium adsorption ratio (PAR), cations, carbonate and bicarbonates were the most effective soil variables which have high significant correlations with the first and second axes. In the upper right side of CCA diagram, the important species (Emex spinosa, Lycium shawii, Bassia indica, Mesembryanthemum nodiflorum and Cynodon dactylon) in group B and Zilla spinosa which was important species in group C were collectively showed a close relationship with sand, cations, sulphate and PAR. While, in the upper left side of the diagram Zygophyllum coccinum which was codominant species in group C, the dominant species (Lavandula coronopifolia) and the important species (Tamarix aphylla, Leptadenia pyrotechnica and Achillea fragrantissima) in group D showed a close relationships with WHC, EC and chlorides. In the lower right side, Avena fatua, Convolvulus arvensis Cynanchum acutum which were important species in group A, Echinops spinosus which was important species in groups B and C showed a close relationship with $\mathrm{HCO}_{3}, \mathrm{pH}$ and organic carbon. In the lower left side, Achillea santolina (dominant species in group A and B), Launaea spinosa (the codominant species in group C), Ochradenus baccatus and Retama raetam (important species in group C) showed close relationships with silt, clay, carbonate and $\mathrm{CaCO}_{3}$.

\section{Discussion}

Achillea (common name, yarrow) is a group of flowering plants in the family Asteraceae described as a genus by Linnaeus in 1753. The genus is native primarily to Europe, temperate areas of Asia, and North America. Achillea grows well in almost any soil type, tolerant of drought, wind and heat. In Egypt occurs in the Oases of western desert, the Mediterranean coastal strip, deserts and Sinai Peninsula [11].

The present study aims at throwing light on investigating the ecological features of two selected species of genus Achillea in family Asteraceae namely, Achillea fragrantissima and A. santolina. The natural plant cover of this study was composed of 74 plant species belonging to 67 genera and related to 23 families. In the Western Mediterranean coast, 27 species are recorded (15 perennials (20.27\%) and 12 annuals (16.21\%)).Similar results were reported in other studies by Ayyad and El-Bayyoumy [25] on the sand dunes of the Western Mediterranean coast and Zahran et al. [26] on vegetation-soil relationship in Sidi Abd El-Rahman coastal lands, while this number is lower than those reported by El-Kady et al. [27] on vegetation of the north-west part of the Nile Delta.

Mashaly [28] stated that after 34 years of Kassas and Zahran [14], there are no fundamental changes neither in

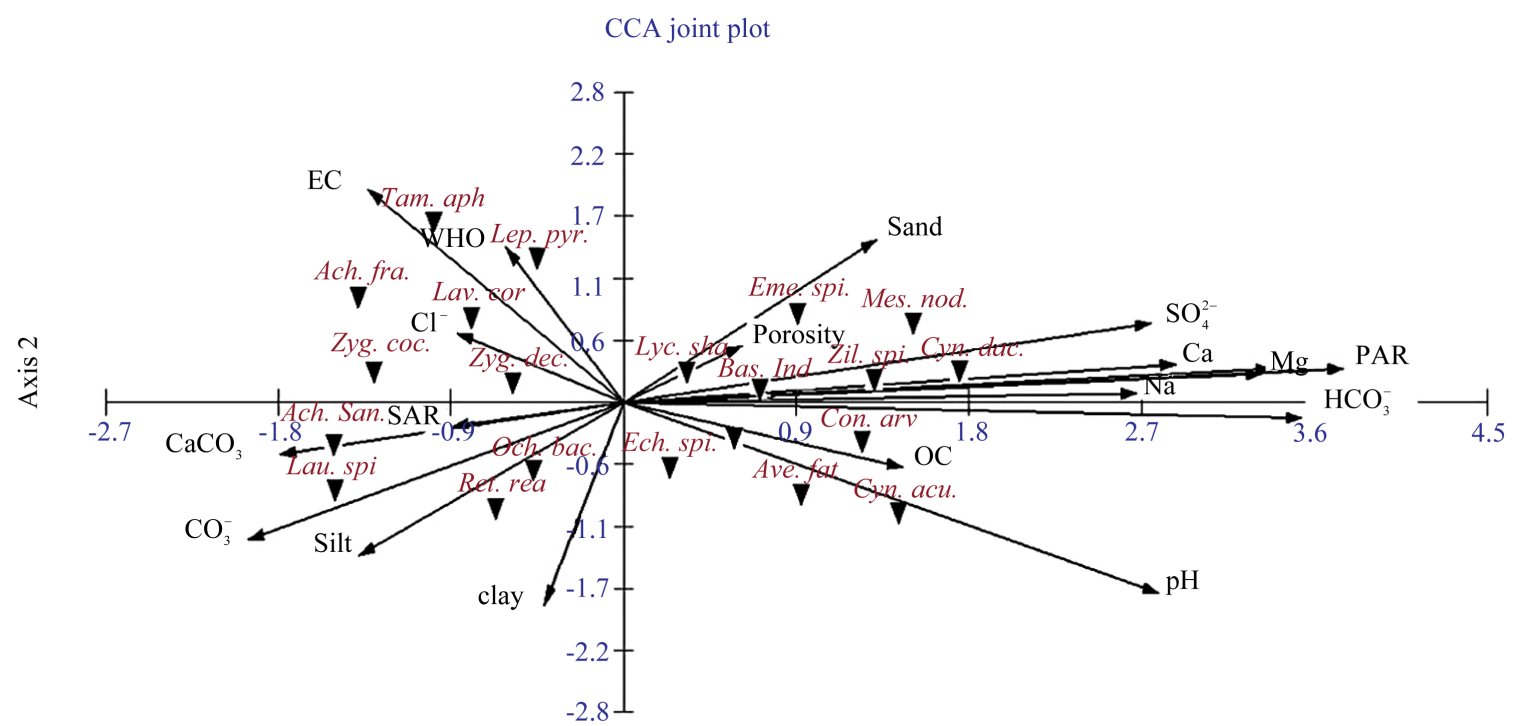

Vector scaling: 4.28

Axis 1

Figure 5. Canonical Correspondence Analysis (CCA) ordination diagram of plant species with soil variables represented by arrows in the study area. The indicator and preferential species are abbreviated to the first four letters of the genus and species respectively. 
the physical environment nor in the vegetation types of Wadi Hagul. Actually, no man interference has been observed in this wadi. In the present study, 57 species are recorded which can be categorized into: 32 perennials (43.24\%), one biennial (1.35\%) and 24 annuals (32.43\%). These results agreed more or less with those investigated by El-Sharkawi et al. [29] on vegetation of inland desert wadies in Egypt, Salama et al. [30] [31] on vegetation analysis in Wadi Qena and floristic composition of the vegetation of Wadi Al-Assiuty and Wadi Habib in the Eastern Desert, respectively, and El-Amier and Abdulkader [32] on vegetation and species diversity in North Galala Desert (Eastern Desert).

Family Asteraceae, Poaceae, Chenopodiaceae, Brassicaceae, Fabaceae and Zygophyllaceae are represented collectively by 48 species ( $64.86 \%$ of the total number of the recorded species). These leading families were reported to be the most frequent in the desert in other investigations by El-Amier et al. [33], Salama et al. [31] and El-Amier et al. [34].

The dominance of perennials (56.75\% of total recorded species) may be related to the nature of the habitat types in the present study in which the reproductive capacity, ecological, morphological and genetic plasticity are the limiting factors [35]. The high contribution of annuals (41.89\% of total recorded species) can be attributed to time of study (April-May 2014) and short life cycle that enables them to resist the instability of the agro-ecosystem [36].

It is worth noting that, the species composition of the studied Western Mediterranean coast varied considerably from the Wadi Hagul (Eastern Desert). This may be attributed mainly to the differences in the nature of soil sediments. The floristic elements of the Western Mediterranean coastal belt enjoy better climatic conditions than those of the other parts of Egypt [37].

Twenty five of the total recorded species are Mediterranean taxa (pluri-, bi- and monoregional). Saharo-Sindian chorotypes, either pure or penetrated into other regions, comprised 47 species (63.51\%) of the total recorded flora. This may reflect the effect of both Mediterranean and Saharo-Sindian chorotypes in the flora of the study area. The presence of the different chorological elements in the study area is believed to be a reflection of intense climatic changes and/or the degradation of the Mediterranean ecosystem which facilitated the invasion of some floristic elements from the adjacent regions [38]. Similar results were reported in other studies [15] [33] [34] [39].

The classification and ordination analyses split the vegetation based on indicator species and their correlation to edaphic factors [39]. The vegetation structure associated with genus Achillea is distinguished by TWINSPAN into four groups. Groups A and B were dominated by Achillea santolina, group C was codominated by Zygophyllm coccinum and Launaea spinosa and group D was dominated by Leptadenia pyrotechnica. Groups A and B may represent the vegetation types of the Western Mediterranean coast of Egypt, while groups C and D may represent the WadiHagul. The identified vegetation groups in the present study were obviously similar to those investigated by Mashaly [28], Abd El-Ghani et al. [39], Zahran et al. [26], Salama et al. [31] and El-Amier et al. [33] [34].

The applying of DCA ordination in the sampled stands in the present study demonstrated that, the four vegetation groups derived by TWINSPAN classification are located on the positive side on the first and second ordination axes. It's obvious that, groups A and B were superimposed at the right side of the diagram, whereas the two remaining groups $\mathrm{C}$ and $\mathrm{D}$ were separated but at the left side of the diagram. The obtained results concerning the DCA ordination in the present investigation were in harmony with the studies of Ramez [40] and Alghanoudi [41].

In the present study linear correlation of soil variables with the importance values of some dominant species indicates significant associations between the floristic composition of the studied area and the edaphic factors such as electrical conductivity, $\mathrm{pH}$, sulphates, bicarbonate, cations $\left(\mathrm{Na}^{+}, \mathrm{K}^{+}, \mathrm{Ca}^{++}\right.$and $\left.\mathrm{Mg}^{++}\right)$and PAR. Moreover, the application of Canoncal Correspondence Analysis (CCA-biplot) between the position of vegetation groups on the ordination planes and soil variables of their stands indicated that, the most important soil factors affecting the distribution of vegetation in the studied areas were: the electrical conductivity, sulphate, $\mathrm{pH}$, calcium carbonate, potassium adsorption ratio (PAR), cations, carbonate and bicarbonates. These results agreed more or less with those investigated by Galal and Fawzy [42], Salama et al. [31], El-Amier and Abdulkader [32].

\section{Conclusion}

From the present work, it could be concluded that the natural cover of the studied areas was composed of 74 
species belonging to 67 genera and related to 23 families. No doubt that the natural vegetation of the Mediterranean coast is changed and suffers from agriculture, urban expansion sand exposed to serious erosion, is of vital importance. Recently, Wadi Hagul (North Galala Desert) is exposed to various factors such as cement factories, mining and the new prepared road which affected its vegetation structure. Therefore, the natural status of the studied area being conservation is of vital importance.

\section{References}

[1] Adriansen, H.K. (2009) Land Reclamation in Egypt: A Study of Life in the New Lands. Geoforum, 40, 664-674. http://dx.doi.org/10.1016/j.geoforum.2009.05.006

[2] Costanza, R., d’Arge, R., de Groot, R., Farberk, S., Grasso, M., Hannon, B., Limburg, K., Naeem, S., O’Neill, R.V., Paruelo, J., Raskin, R.G., Sutton, P. and van den Belt, M. (1997) The Value of the Ecosystem Services and Natural Capital. Nature, 387, 253-260. http://dx.doi.org/10.1038/387253a0

[3] Balmford, A., Bruner, P., Cooper, R., Costanza, S., Farber, R.E., Green, M., Jenkins, P., Jefferiss, V., Jessamy, J., Madden, K., Munro, N., Myers, S., Naeem, J., Paavola, M., Rayment, S., Trumper, K. and Turner, R.K. (2002) Economic Reasons for Conserving Wild Nature. Science, 297, 950-953. http://dx.doi.org/10.1126/science.1073947

[4] Heneidy, S.Z. and Bidak, L.M. (2004) Potential Uses of Plant Species of the Costal Mediterranean Region, Egypt. Pakistan Journal of Biological Sciences, 7, 1010-1023. http://dx.doi.org/10.3923/pjbs.2004.1010.1023

[5] Täckholm, V. (1974) Students’ Flora of Egypt. 2nd Edition, Cairo University Publishing, Beirut, 888.

[6] Boulos, L. (1995) Flora of Egypt: A Checklist. Al-Hadara Publishing, Cairo.

[7] Boulos, L. (1999, 2000 \&2005) Flora of Egypt: Vol. 1, 2, 4. Al-Hadara Publishing, Cairo.

[8] Zahran, M.A. and Willis, A.J. (2009) The Vegetation of Egypt. 2nd Edition, Springer, Netherlands.

[9] Ozenda, P. (1983) Flore du Sahara. En Editions du Centre National de la Recherche Scientifique (CNRS), Paris, 21-32.

[10] Boulos, L. (2002) Flora of Egypt: Vol. 3. Al-Hadara Publishing, Cairo.

[11] Boulos, L. (2009) Flora of Egypt. Checklist All-Hadara Publishing, Cairo.

[12] UNESCO/FAO (1963) Bioclimatic Map of the Mediterranean Zone. Explanatory Notes, Arid Zone Research, 2217 p.

[13] Ayyad, M.A. and Hilmy, S.H. (1974) The Distribution of Asphodelus Micro-Carpus and Associated Species on the Western Mediterranean Coast of Egypt. Ecology, 55, 511-524. http://dx.doi.org/10.2307/1935143

[14] Kassas, M. and Zahran, M.A. (1962) Studies on the Ecology of the Red Sea Coastal Land, I. The District of Gebel Ataqa and El-Galala El-Bahariya. Bulletin de la Société de Géographie d'Egypte, 35, 129-175.

[15] Kassas, M. and Zahran, M.A. (1965) Studies on the Ecology of the Red Sea Coastal Land, II. The District from El-Galala El-Qibliya to Hurghada. Bulletin de la Société de Géographie d'Egypte, 38, 155-193.

[16] Raunkiaer, C. (1934) The Life Forms of Plants and Statistical Plant Geography. Oxford University Press, London.

[17] Shukla, R.S. and Chandel, P.S. (1989) Plant Ecology and Soil Science. S. Chand \& Company LTD. Ram Nagar, New Delhi.

[18] Canfield, R. (1941) Application of the Line Interception Method in Sampling Range Vegetation. Journal of Forestry, 39, 288-394.

[19] Piper, C.S. (1947) Soil and Plant Analysis. Intersience Publishers, Inc., New York.

[20] Jackson, M.L. (1962) Soil Chemical Analysis. International Institute for Tropical Agriculture (IITA), Manual Series No.1, 70 p.

[21] Allen, S.E., Grimshaw, H.M., Parkinson, J.A., Quarmby, C. and Roberts, J.D. (1974) Chemical Analysis of Ecological Materials. Blackwell Scientific Publications, Osney, Oxford and London.

[22] Hill, M.O. and Šmilauer, P. (2005) TWINSPAN for Windows Version 2.3. Centre for Ecology and Hydrology, University of South Bohemia, Huntingdon \& České Budějovice.

[23] ter Braak, C.J. (1987) The Analysis of Vegetation-Environment Relationship by Canonical Correspondence Analysis. Vegetatio, 69, 69-77. http://dx.doi.org/10.1007/BF00038688

[24] ter Braak, C.J. (1988) CANOCA-A FORTRAN Program for Canonical Community Ordination by Partial Detreded Correspondence Analysis, Principal Component Analysis and Redundancy Analysis (Version 2.1). Agric. Math. Group, Wageninigen.

[25] Ayyad, M.A. and El-Bayyoumi, M.A. (1979) On the Phytosociology of Sand Dunes of the Western Desert of Egypt. In: Singh, J.S. and Gopal, B., Eds., Glimpses of Ecology, International Scientific Publications, Jaipur, 219-237.

[26] Zahran, M.A., Ahmed, A.M., Abd El-Gawad, A.M. and Shawky, R.A. (2013) Vegetation Soil-Relationship of Medi- 
terranean Coastal Desert ar Sidi Abd El-Rahman Area, Egypt. Egyptian Journal of Botany, 53, 305-317.

[27] El-kady, H.F., Shaltout, K.H., El-Shourbagy, M.N. and Al-sodany, Y.M. (2000) Characterization of Habitats in the North Western Part of the Nile Delta. Proceedings of the 1st International Conference on Biological Sciences, Tanta, 7-8 May 2000, 144-157.

[28] Mashaly, I.A. (1996) On the Phytosociology of Wadi Hagul, Red Sea Coast. Egyptian Journal of Environmental Sciences, 12, 31-54.

[29] El-Sharkawi, H.M., Salama, F.M. and Fayed, A.A. (1982) Vegetation of Inland Desert Wadies in Egypt III. Wadi Gimal and Wadi El-Miyah. Feddes Repertorium, 93, 135-145. http://dx.doi.org/10.1002/fedr.19820930117

[30] Salama, F.M., Ahmed, M.K., El-Tayeh, N.A. and Hammad, S.A. (2012) Vegetation Analysis, Phenological Patterns and Chorological Affinities in Wadi Qena, Eastern Desert, Egypt. African Journal Ecology, 50, 193-204. http://dx.doi.org/10.1111/j.1365-2028.2011.01313.x

[31] Salama, F., El-Ghani, M.A., Gadallah, M., Salah, E.N. and Ahmed, A.M. (2014) Variations in Vegetation Structure, Species Dominance and Plant Communities in South of the Eastern Desert-Egypt. Notulae Scientia Biologicae, 6, 4158.

[32] El-Amier, Y.A. and Abdulkader, O.M. (2015) Vegetation and Species Diversity in the Northern Sector of Eastern Desert, Egypt. West African Journal of Applied Ecology, 23, 75-99.

[33] El-Amier, Y.A., El-Halawany, E.F. and Abdullah, T.J. (2014) Composition and Diversity of Plant Communities in Sand Formations along the Northern Coast of the Nile Delta in Egypt. Research Journal of Pharmaceutical, Biological and Chemical Sciences, 5, 826-847.

[34] El-Amier, Y.A., Haroun, S.A., El-Shehaby, O.A. and Abdulkader, O.M. (2015) Floristic Features of Northern Sector of the Eastern Desert, Egypt. Journal of Environmental Sciences, 44, 387-401.

[35] Grime, J.P. (1979) Plant Strategies and Vegetation Processes. John Wiley \& Sons, Chichester.

[36] Sans, F.X. and Masalles, R.M. (1995) Phenological Patterns in an Arable Land Weed Community Related to Disturbance. Weed Research, 35, 321-332. http://dx.doi.org/10.1111/j.1365-3180.1995.tb01627.x

[37] Zahran, M.A. and Willis, A.J. (1992) The Vegetation of Egypt. Chapman \& Hall, London, 424. http://dx.doi.org/10.1007/978-94-015-8066-3

[38] Madi, M.I., Shaltout, K.H. and Sharaf El-Din, A. (2002) Flora of the Coastal Sand Dunes of Gaza Strip, Palestine. Proceedings of the 2nd International Conference on Biological Sciences, Tanta, 27-28 April 2002, 64-78.

[39] Abd El-Ghani, M.M., Soliman, A., Hamdy, R. and Bennoba, E. (2013) Weed Flora in the Reclaimed Lands along the Northern Sector of the Nile Valley in Egypt. Turkish Journal of Botany, 37, 464-488.

[40] Ramez, B. (2015) Ecological Study and Economic Potentialities of Some Species of Genus Launaea in Egypt. Master's Thesis, Faculty of Science, Mansoura University, Mansoura.

[41] Alghanoudi, G.A. (2015) Non-Conventional Fiber Resources from Plants Naturally Growing in Different Habitats of Egypt. Master’s Thesis, Faculty of Science, Mansoura University, Mansoura.

[42] Galal, T.M. and Fawzy, M. (2007) Sand Dune Vegetation in the Coast of Nile Delta, Egypt. Global Journal of Environment Research, 2, 74-85. 